\title{
CHAPTER 3.5
}

\section{UNCERTAINTIES AND UNKNOWNS IN ATMOSPHERIC OBSERVATIONS: HOW DO THEY AFFECT THE SOLAR SIGNAL IDENTIFICATION?}

\author{
Bernd Funke $^{1}$, Annika Seppälä ${ }^{2}$ and Thomas von Clarmann ${ }^{3}$
}

\section{Introduction}

A large variety of observations, used to identify the solar fingerprint on Earth's atmosphere, have been discussed in the previous chapters. All of them have limitations and uncertainties which have to be considered in their analysis. The main difficulties in the identification of solar signals from atmospheric measurements are related to three principal observational limitations:

- Instrument precision and resolution. Solar-induced variations of atmospheric observables are often too small to be resolved within the instrumental precision. Also, drifts may arise from instrumental degradation over longer time periods.

- Temporal coverage of observational records. Particularly satellite data, being of highest utility for solar signal detection on a global scale, are available only for the last three decades, a period too short for an unequivocal attribution of observed signals to mid- and long-term solar variability.

- Interference with other sources of variability (see Chapter 4.3). Atmospheric variability is typically dominated by other sources than solar variability. The extraction of the solar signal is thus often challenging, particularly if other sources of variability show an apparent correlation with solar activity.

The relevance of the latter two limitations depends on the timescale of solar activity variations. Transient solar events such as solar proton events produced

\footnotetext{
1 Instituto de Astrofísica de Andalucía, CSIC, Granada, Spain

2 Earth Observation Unit, Finnish Meteorological Institute, Helsinki, Finland

${ }^{3}$ Karlsruhe Institute of Technology, Institute for Meteorology and Climate Research, Karlsruhe, Germany
} 
by solar flares leave a clear fingerprint in the chemical composition of the atmosphere. In such cases, the attribution of the atmospheric changes to a solar event is relatively easy. Similarly, the response of several short-lived trace gases (such as atomic oxygen and nitric oxide in the upper mesosphere and lower thermosphere) to the 27-day solar rotation can be unequivocally identified by time series analysis from observational records, provided the signal is large enough and the temporal coverage is sufficiently long to provide a statistically robust dataset.

The situation is much more complicated for the Schwabe cycle, which affects the atmosphere by solar irradiation variations and by the modulation of energetic particle fluxes. Atmospheric responses are small and generally masked by the background variability. Furthermore, long-term datasets covering at least one full Schwabe cycle (ideally several cycles) are required. However, such datasets are often not available. Direct chemical responses to solar variations (e.g., the ozone response in the tropical upper stratosphere to solar irradiance variations in the ultraviolet or the reactive nitrogen response in polar winter to particle flux variations) are the most promising candidates to search for Schwabe cycle signals in observations, as they follow directly the solar forcing variations. Indirect responses occurring by transport of processed air masses and circulation changes in response to solar activity are more difficult to detect because of the time delays introduced by these coupling mechanisms. Particularly in the latter case, interferences with internal atmospheric variability drivers (a problem known as aliasing) are a major issue in time series analysis of both observational and model data. As an example, a recent study (Chiodo et al., 2014) suggests that a large fraction of the apparent solar-induced lower stratospheric temperature and ozone response, inferred by means of multilinear regression analysis of a 44-year model simulation, could be due to two major volcanic eruptions (i.e., El Chichón in 1982 and Mt. Pinatubo in 1991), which are concurrent with periods of high solar activity (see Figure 1).

Each type of observation is confronted with different problems and limitations, which will be discussed separately in the following sections. Hence, it is clear that the accurate solar signal identification in the atmosphere benefits form the synergistic use of multiple observation techniques.

\section{Proxy-based observations}

In the context of this chapter, we refer to proxies as preserved physical characteristics of the past that stand in for direct measurements, to enable scientists to reconstruct the atmospheric conditions that prevailed during much of the Earth's history (see also Chapter 2.1). Proxy-based observations, such as temperature reconstructions from tree rings or nitrate depositions in ice cores, the latter potentially providing information on atmospheric ionisation by cosmic rays, are used to identify solar signals on very long time scales.

However, the principal problem of these reconstructions is that the relationship of the proxies with the respective atmospheric parameters can only be established at present when direct observations of the latter are available. A drift or 


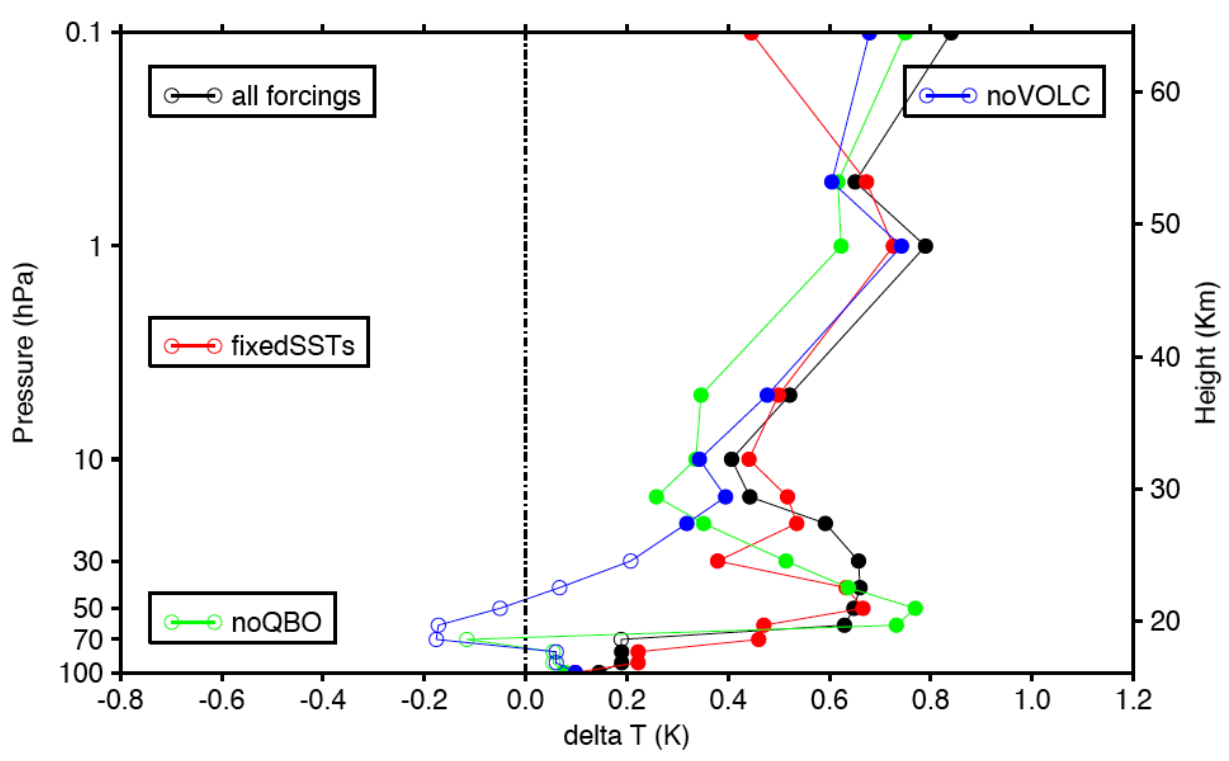

Fig. 1. Solar signal (response to a $2-\sigma$ variation of the $240-270 \mathrm{~nm}$ UV flux, corresponding to about $70 \%$ of the average Schwabe cycle variation) in the tropical zonal mean temperature as estimated from multiple linear regression analysis of a 44-year climate model simulation including all non-solar forcings (all forcings), assuming fixed sea surface temperatures (fixed SSTs), as well as excluding the quasi-biennal oscillation (no QBO) and volcanic eruptions (no VOLC). Note that the modelled lower stratospheric solar signal vanishes when excluding volcanic eruptions. Reprinted from Chiodo et al. (2014).

modulation of this relationship by other climate forcings (e.g., volcanoes or variations in green house gas concentrations) and by variations of the Earth's magnetic field over longer historical periods cannot be excluded. This is particularly important if such modulations show a correlation with the solar signal to be extracted.

\section{In situ observations}

In situ instruments such as radiosondes provide very accurate observations of tropospheric and lower stratospheric meteorological parameters potentially affected by solar variability. Some in situ observations (e.g, surface temperature and sea level pressure measurements) have been made routinely for more than a century.

However, these local measurements are often not representative for the atmospheric mean state even on a regional scale. Therefore, data assimilation techniques (see Chapter 3.4) are required to reconstruct global synoptic fields from a large amount of individual measurements. Furthermore, both the accuracy and the geographic coverage of historic data are generally not comparable to 
modern standards, making the analysis of long-term time series with respect to solar signals highly challenging. Evidently, it is much more difficult to obtain in situ observations in the middle atmosphere. High-altitude balloons and rockets have been used for stratospheric and mesospheric air sampling only on a sporadic basis during the last decades. Due to their sparseness, these measurements are of limited use for solar signal detection in the middle stratosphere and mesosphere.

\section{Remote sensing observations}

Remote sensing techniques allow to infer many atmospheric parameters (i.e., temperature, composition, clouds, etc.) by observing the radiance emitted, transmitted or scattered by the Earth's atmosphere from a remote location. This radiance modulation is taking place over the entire light path observed by the instrument. Remote sensing observations are thus integrated over large air volumes, but the information on small-scale structures is generally lost. In contrast to in situ observations, they only provide an indirect measure of the observables of interest, because the latter have to be inferred from the observed radiances by inverting the radiative transfer equation.

\subsection{Atmospheric soundings from the ground}

Ground-based remote sensing instruments (see Chapter 3.2) provide long-term observations of temperature, trace gases, and cloud parameters, potentially affected by solar variability. For example, ground-based total ozone column observations are available since the beginning of the last century. Calibration and substitution of individual instruments is much easier on the ground than in space, guaranteeing stable long-term records that are required for the detection of decadal variability related to solar variations. Nowadays, global networks of inter-calibrated groundbased instruments provide measurements in all continents and climate zones.

However, the limited vertical resolution of some ground-based instruments, resulting from the upward looking geometry, complicates the identification of altitude-dependent solar responses, as in the case of tropical stratospheric ozone.

\subsection{Satellite observations}

Space instruments (see Chapter 3.3) provide observations of atmospheric key parameters with a large spatial coverage. Hence, they are most suited for solar signal detection on a global scale. However, atmospheric observations from space are available only since the late 1970s, covering less than three Schwabe cycles. Moreover, the typical lifetime of space missions does rarely exceed 5-10 years. Long-term observational records, required for the detection of Schwabe cycle signals, can therefore only be obtained by combining data from multiple instruments.

Since inter-calibration of different satellite sounders is much more complicated than that of ground-based instruments, the construction of multi-instrument climate records is not straightforward and requires complex mathematical tools. 

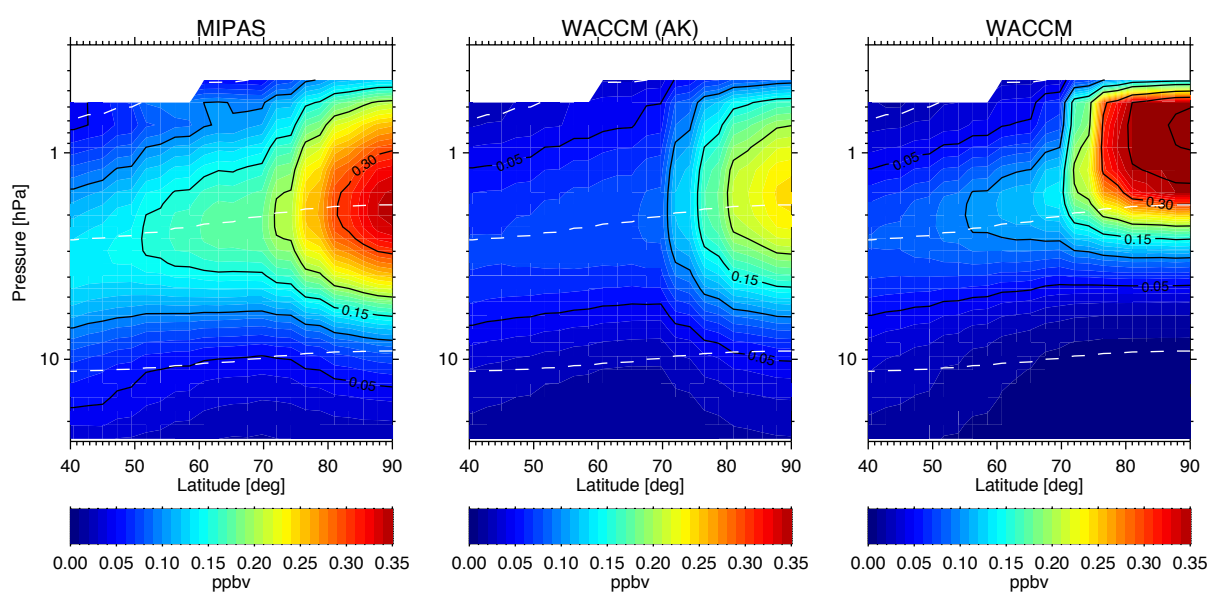

Fig. 2. Observed (left) and modelled (middle) hypochlorous acid ( $\mathrm{HOCl}$ ) response to the October/November 2003 solar proton event in the Arctic polar stratosphere. Only after taking into account the instrument response function by applying MIPAS averaging kernels to the model data (middle), the vertical structures of observed and modelled responses become comparable and a slight underestimation of the model result shows up. Reprinted from Funke et al. (2011).

The temporal overlap of successive equivalent instruments is crucial and has to be taken into account in the planning of space missions. The continuity of satellite data records in the future is vital to the understanding of the atmospheric response to solar variations on a longer time scale. Current space missions targeting stratospheric and mesospheric observations have ended recently, e.g., ENVISAT, or will be phased out over the next few years. As a consequence, an observational gap is expected in the second half of this decade, which will seriously harm the continuity of observational long-term records.

For some space instruments, the sampling pattern is not uniform, which causes additional complication (i.e., sampling errors, see Toohey et al. (2013)). This is particularly true for solar occultation instruments, which can measure only during local sunrise or sunset, or for instruments in a slowly drifting orbit. The latter is of particular importance if the orbit characteristics change on timescales comparable to the Schwabe cycle.

In contrast to nadir-sounders, which look from the satellite through the atmosphere to Earth's surface, limb emission, limb scattering and occultation instruments look horizontally through the atmosphere. By varying the viewing angle, a good altitude resolution of the signal can be obtained and vertically-structured solar signals in temperature and composition can be extracted from the measurements.

However, remote sensing instruments, in contrast to in situ instruments, always provide a smeared or even distorted picture of reality. This deviation can be 


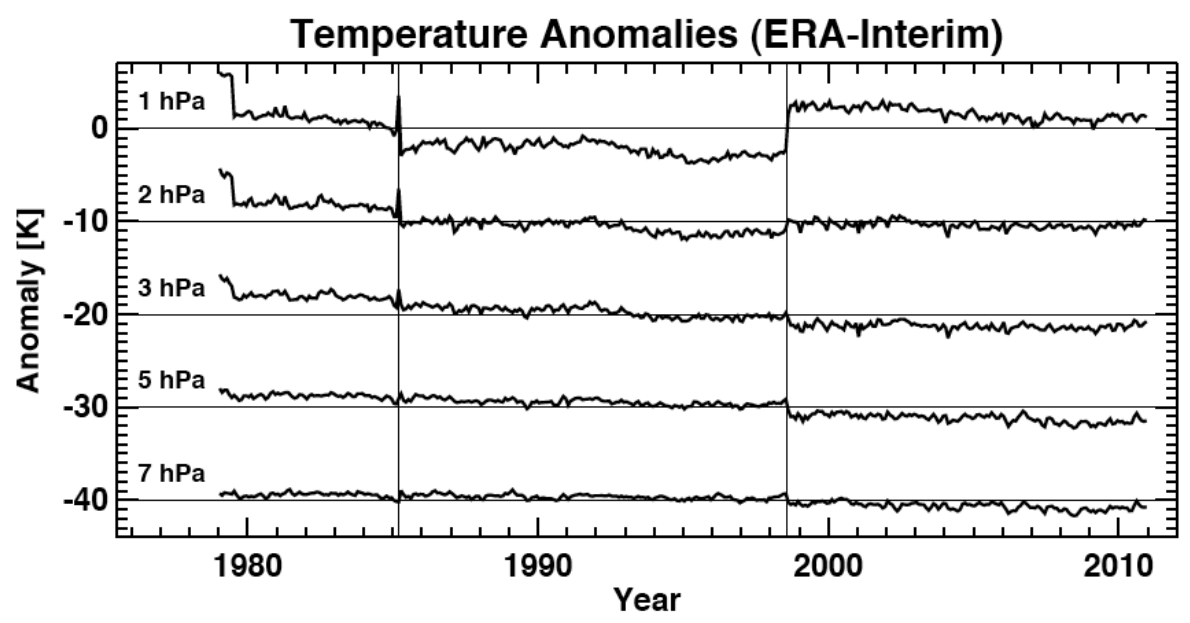

Fig. 3. Global and monthly mean ERA-Interim temperature anomalies due to changes in satellite radiance data used in the assimilation at the top five pressure levels in the stratosphere. The curves at 2, 3, 5 and $7 \mathrm{hPa}$ are shifted by $10 \mathrm{~K}$ with respect to the curve above. The jump times in March 1985 and August 1998 are denoted by the thin vertical lines. Reprinted from McLandress et al. (2014).

taken into account when measured data are compared to data simulated by climate models by application of the instrument response function, expressed by the so-called averaging kernel (Rodgers, 2000), to the model data. This is particularly important for nadir measurements with their limited altitude resolution, but can also be important for the better-resolving limb sounders. Funke et al. (2011) compared $\mathrm{HOCl}$ measurements from the MIPAS limb emission spectrometer to model results and found large discrepancies which could be removed by the application of the grossly asymmetric MIPAS averaging kernel which shifted the maximum of the concentration profile (see Figure 2).

\section{Re-analysis data}

Consistent distributions of pressure, temperature, humidity, trace gas concentrations and others are compiled in the so-called 're-analysis data' (see Chapter 3.4), which are obtained by assimilation of observed data in numerical weather prediction (NWP) models. Observations used in data assimilation cover a wide range of in situ, ground-based, and satellite data. In this sense, the re-analysis of assimilated meteorological and chemical fields is a powerful tool to make use of complementary information derived from different observational techniques in a synergistic manner.

On the other hand, it is evident that all limitations and uncertainties encountered in the observations will propagate into the re-analysis data products. Also, 
discontinuities may arise when new instruments are included into the data assimilation system (see Figure 3), which may lead to artefacts in time series analysis with respect to solar signals. An additional problem arises from the influence of the NWP model itself, which might suffer from an incomplete or erroneous representation of atmospheric processes involved in solar signal propagation. Particularly at high altitudes, where observational data is sparse, this can lead to important deviations of solar responses in the re-analysis data.

\section{Further reading}

Rodgers, C. D. 2000, Inverse Methods for Atmospheric Sounding: Theory and Practice, World Scientific Publishing Co. Pte. Ltd, Singapore.

Toohey, M. et al. 2013, Characterizing sampling biases in the trace gas climatologies of the SPARC Data Initiative, J. Geophys. Res. (Atmospheres), 118, 11847-11862.

Wilks, D. S. 2011, Statistical Methods in the Atmospheric Sciences: an Introduction, International Geophysics. Academic Press, San Diego, 3rd edition. 
